Available online at http://www.mecs-press.net/ijeme

\title{
The on-line continuous casting slab surface quality prediction expert system
}

\author{
Jin Yan $^{\mathrm{a}}$ Bi Xuegong ${ }^{\mathrm{b}}$ Tian Zhibing $^{\mathrm{c}}$ \\ Key Laboratory for Ferrous Metallurgy and Resources Utilization of Ministry of Education \\ Wuhan University of science and technology Wuhan, P. R. China
}

\begin{abstract}
An on-line continuous casting slab surface quality prediction expert system is developed. The developing work is nearly completed and it may be the first system of the relating field in China. The main structure of the online continuous casting slab surface quality prediction expert system is that the slab surface quality is predicted online with expert system, and the system can alarm breakout in continuous casting with BP neural net and logic methods, based on the collected thermal couple data in mould and the process parameter transferred from the Continuous Casting machine server.
\end{abstract}

Index Terms: expert system; continuous casting; slab; quqlity control

(C) 2012 Published by MECS Publisher. Selection and/or peer review under responsibility of the International Conference on E-Business System and Education Technology

\section{Introduction}

In modern steel-making industry the quality requirements of the product are increasingly complex [1-3]. So it is expected that the product quality can be predicted on-line during the producing stage, such as, the slab surface quality prediction expert system [4-9].

So, an on-line continuous casting slab surface quality prediction expert system is developed. The developing work is nearly completed and it may be the first system of the relating field in China.

Corresponding author:

E-mail address: ${ }^{j}$ jinyan203558@yahoo.com.cn , ${ }^{b}$ xuegong@public.wh.hb.cn, ${ }^{c}$ yejinxixuesheng@163.com 


\section{The main structure of the system}

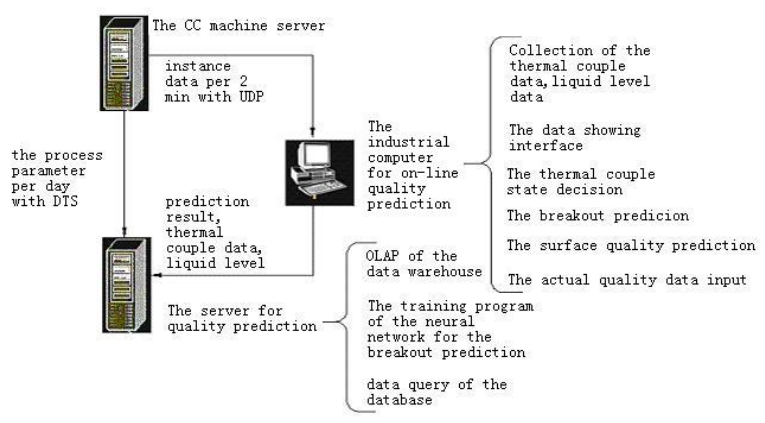

Figure 1. The structure of the on-line expert system

The main structure of the on-line continuous casting slab surface quality prediction expert system is that the slab surface quality is predicted online with expert system, and the system can alarm breakout in continuous casting with BP (Back Propagation) neural net and logic methods, based on the collected thermal couple data in mould and the process parameter transferred from the CC (Continuous Casting) machine server. The structure of the system is shown in Fig. 1.

The hardware of the system includes: a data warehouse server for data support, a industrial computer for quality prediction, 10 ADAM 4018 modules for data collecting, and a ADAM 4520 module for data communication.

The software of the system includes:

- $\quad$ system software: Windows 2000 Server,

- data base software: SQL Server 2000,

- $\quad$ programming language: Visual Basic 6.0,

- $\quad$ expert system tool language: CLIPS 6.2.

\section{The data warehouse of the slab quality data}

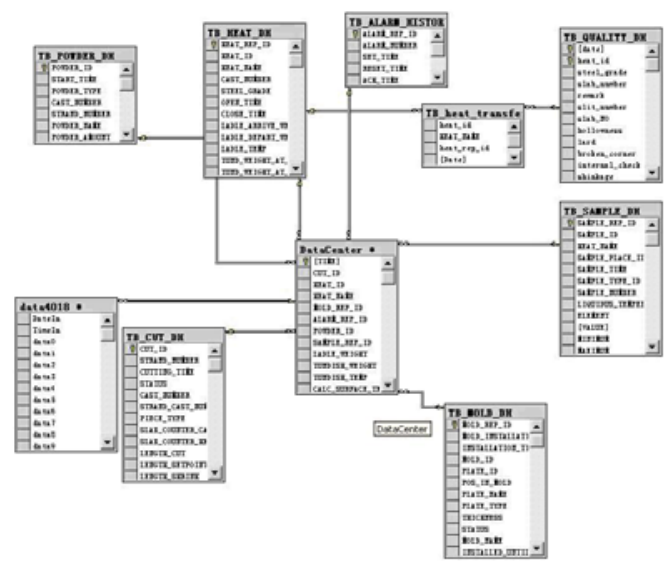

Figure 2. The structure of the on-line expert system 
The data in the system is achieved in data warehouse developed with SQL Server 2000. The decision tree and clustering type data mining technologies are used to find out the rule in expert system. There are two buffer databases in the data ware house, in order to complete the data integration. The data are collected from the thermal couple data and liquid steel level data, and are also transferred from the continuous casting machine with UDP (User Datagram Protocol) Server and DTS (Data Transformation Services) of SQL server 2000. In addition, the quality data is inputted from Visual Basic interface by human being.

The data from data base in the continuous casting machine server can be selected with data warehouse and the data is transferred into the data warehouse. However, the data in the continuous casting machine server are not integrated. The data integration is a hard work. In the design of the data link mode in the data warehouse, at first the star mode is concerned, but it is found that the continuous casting process is complex, and the data source is complex, so the snow-flake mode is taken as the data link mode in the data warehouse, Fig. 2. The data is classified by heat id and slab id.

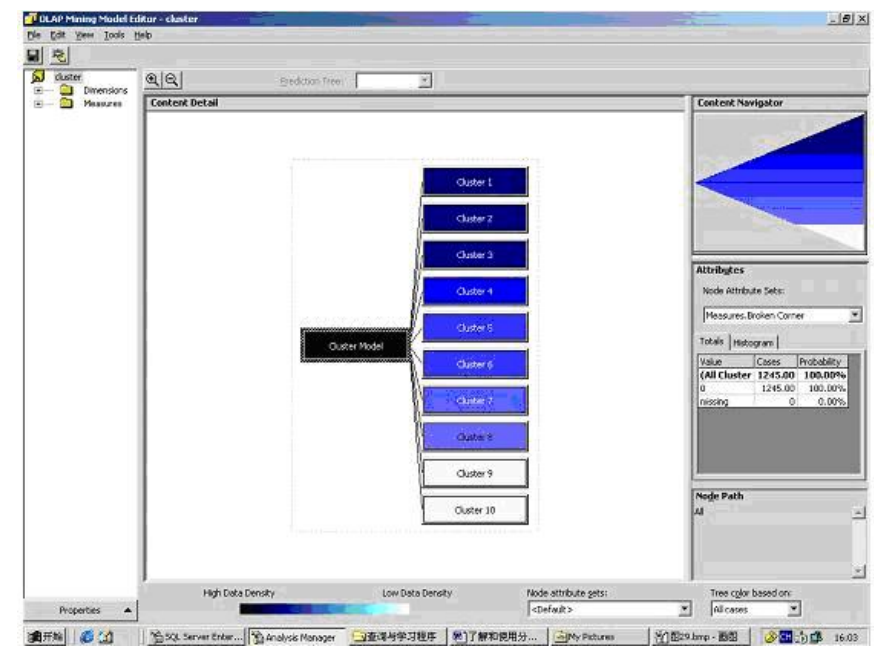

Figure 3. The structure of the on-line expert system

There is data analysis service in SQL Server 2000. Before the data analysis, the data cube should be created, and based on cube the data should be treated, the data in the data warehouse can be drilled, i.e. the data source can be outputted. In addition, the data in the data warehouse can be mined with Microsoft decide tree model and Microsoft clustering model. For example, one of the data mining results with Microsoft clustering model is that if casting speed is less then $0.5 \mathrm{~m} / \mathrm{min}$, and water temperature in the mould is between 34 and $41^{\circ} \mathrm{C}$, slab would have defect of corner cracking, Fig.3. One of the data mining with Microsoft decide tree model is that if the No.2 water table is used in the secondary cooling section, the slab would have defect of surface longitudinal cracking.

The data transfer technologies in the data warehouse include UDP technology, DTS technology in SQL Server 2000, and the human being input technology.

\section{Slab surface quality prediction expert system}

The surface quality prediction expert system is developed with CLIPS language. The knowledge sources include the experience knowledge in site of CC machine, the reference knowledge from relating article and knowledge from data mining. All the knowledge is transferred to rules. The reasoning machine is production rule system, and the reasoning strategy is stack and salience combined methods. The fact base of the CLIPS 
system include the thermal couple data facts, the process parameter facts, the thermal couple working state facts, the thermal couple temperature mode facts and quality predict facts. CLIPS system can be combined into Visual Basic with DLL. The data transfer between the two languages is using the file type database. The expert system running cycle is $2 \mathrm{~min}$, and it can provide the alarm of recent surface quality defect and the operation suggesting of CC machine.

In the knowledge base, the number of the knowledge and the express method of the knowledge affect the ability of the expert system to a large extent. The knowledge acquisition and the knowledge express way are the key part of the knowledge base building. In the theory of expert system, the knowledge acquisition is the most difficult part in the developing of expert system. Based on the characteristic of the system, the knowledge can be obtained from the experience knowledge and the mechanism knowledge. The experience knowledge includes the experience of the mould surface quality prediction, caster operation and the thermal couple working state etc. The mechanism knowledge includes the mechanism of slab surface defect etc.

In CLIPS there are three knowledge express methods, i.e. rule, process program and the object-oriented program. According to the characteristics of the slab surface quality prediction expert system, the system is designed as the rule production system. After the knowledge acquisition, the knowledge is transferred to rule according to the CLIPS language specification. In the knowledge base there are initializing rules, anti-error rules, and prediction decide rules. The initializing rules provides the initial fact for the knowledge base when the system starting up. The anti-error rules check the input data. The predictions decide rules can reason the knowledge of the slab surface quality prediction.

The reasoning engine is the acting part of the system. According to the characteristics of the system, the system is designed as the forward chain reasoning, and the parts in the reasoning engine include mode-matching algorithm, conflict resolution strategy, and rule acting etc. The mode-matching algorithm is that every rule is checked and a group of facts is found to satisfy the rule. If it is found, the rule would be put into the agenda, and if the rule is acted, the facts in the fact base would be changed. The conflict resolution strategy is the selecting strategy of the facts in the agenda. The strategy is the stack and salience combined methods. The rule acting is that the right part of the rule in the agenda is acted, after the CLIPS run command acting. And the predicting results are obtained.

The data in the fact base is the base on which the quality prediction is acted. The thermal couple data in mould and caster process data are stacked in the fact base. Before the facts are created, the slot in the facts should be defined. And then, the quality prediction fact base is created.

As the other components of the continuous casting slab surface prediction expert system is programmed with Visual Basic 6.0, the CLIPS program should be inserted into the Visual Basic. So, the DLL of CLIPS for Visual Basic is programmed. The Visual Basic uses the API function to act the CLIPS program. The CLIPS program uses the data in the data base and output the results to the database for the VB program. In this way the CLIPS program acting cycle is $2 \mathrm{~min}$. The expert system is running stable and liable, and is a on-line expert system.

\section{Conclusions}

The slab surface prediction expert system for the continuous caster is developed with little cost. It has the following features:

- The expert system in the quality prediction system is developed with CLIPS6.2 language, which is with the characteristics of high portability, low cost, and ease of integration with external systems.

- The expert rules in the system come from operation experiences and are tested with the mathematic models.

- The breakout prediction methods in the system include neural network method and logic method.

- The data in the system is stocked in the data warehouse. The data in the data warehouse can be mined to establish the expert rules. The abnormal data can be found in a large quantity of data with the OLAP tools of the SQL Server 2000. 


\section{References}

[1] H.Preissl, T.Faster, “Automatic quality control of cast slabs at the VOEST-ALPINE Steel works”, Steelmaking Conference Proceedings, Pittsburgh, USA, vol. 78, pp. 621-627, March 1995.

[2] A.S.Normanton, P.N.Hewitt, N.S. Hunter et. al., "Mould thermal monitoring: a window on the mould", Ironmaking and Steelmaking, UK, vol. 31, pp.357-363, May 2004.

[3] Ir. F Hears, S. G. Thorton, "The application of mould thermal monitoring on the two-strand slab caster at Sidmar”, Steelmaking Conference Proceedings, Pittsburgh, USA, vol. 77, pp. 390-398, March 1994.

[4] M. M. Collur, B. V. Patil and M. J. Korzi, "Evaluation of the Mannesman Demag mold thermal monitoring system at the A-L's Brackenridge slab caster”, Steelmaking Conference Proceedings, Pittsburgh, USA, vol. 83, pp. 413-432, March 2000.

[5] A. S. Normanton, B. Barber, A. Bell, et. al., "Developments in online surface and internal quality forecasting of continuously cast semis”, Ironmaking and Steelmaking, UK, vol. 31, pp.376-382, May 2004.

[6] J. Schwedmann, J. Wochnik, "Instrumentation system, automation control and quality control for products of the continuous casting process", The second international conference on continuous casting of steel, Wuhan, China, vol. 2, pp. 356-360, May 1995.

[7] P.Bellomo, G. Salvemini, E. S. Maria, et. al., “Online detection of mold thermal transfer characteristics”, Steelmaking Conference Proceedings, Pittsburgh, USA, vol. 77, pp. 318-325, March 1994.

[8] S. Kumar, "Development of intelligent mould for online detection of defects in steel billets", Ironmaking and Steelmaking, UK, vol. 26, pp.268-273, April 1999.

[9] W. H. Emling, S. Daoson, “Mold instrumentation for breakout detection and control”, Steelmaking Conference Proceedings, Pittsburgh, USA, vol. 77, pp. 196-201, March 1991. 